\title{
Developing key performance indicators to measure the progress of regional regulatory convergence and cooperation in Asia-Pacific Economic Cooperation (APEC)
}

\author{
Sannie Siaw Foong Chong ${ }^{1 *}$, John C. W. Lim² and Toshiyoshi Tominaga ${ }^{3}$
}

\begin{abstract}
Regulatory convergence and cooperation among the authorities in the field of medical products (medicines and medical devices) are essential to deliver safe and efficacious products quickly to patients. APEC established the Regulatory Harmonization Steering Committee (RHSC) to advance this cause among member economies. This paper identifies four areas of appropriate regulatory practice in which APEC economies could converge, and explores the feasible processes of how APEC economies could cooperate in order to bring about realization of a maximum level of regulatory convergence by 2020.
\end{abstract}

Keywords: APEC, Regulatory convergence and cooperation, Key performance indicators

\section{Background}

Since the inception of the International Council for Harmonisation of Technical Requirements for Pharmaceuticals for Human Use (ICH) in 1990, harmonization of regulatory requirements has been progressively and successfully achieved in major jurisdictions around the world. This has benefited global public health and national health systems mainly through transparency and predictability of what scientific dataset is required to support the approval of a new pharmaceutical, hence advancing the innovation of drug development. In addition, convergence and cooperation (information exchange, work sharing, reliance, and recognition) (WHO Good Regulatory Practices 2016) have been facilitated among the ICH regulatory members (ICH Official website 2018) in order to optimize efficiency in regulatory processes without duplication in efforts.

In 2010, the RHSC established under APEC's Life Sciences Innovation Forum (LSIF) advocated for regulatory convergence in pharmaceuticals for improved public

\footnotetext{
* Correspondence: sannie.chong@roche.com

${ }^{1}$ Asia Pacific Technical Regulatory Policy, Pharma Technical Regulatory Policy and International Operations, Roche Singapore Technical Operations, F. Hoffmann La-Roche Ltd, Singapore, Singapore

Full list of author information is available at the end of the article
}

health and economic development among its 21 economies (APEC Member Economies 2017). Convergence will focus on the process of aligning multiple countries' regulations for greater regulatory cooperation and does not necessarily require the regulations to be "harmonized". According to an RHSC strategic framework document released in 2010, while each economy may adopt each phase on its own timeframe, the ultimate aim would be for APEC economies to achieve the maximum level of regulatory convergence feasible by 2020 (APEC RHSC Vision 2020).

This paper identified four areas of appropriate regulatory practice in which APEC economies could converge. The four areas are related to the use of (1) Certificate of Pharmaceutical Product (CPP); (2) the Pharmaceutical Inspection Co-operation Scheme (PIC/S) membership; (3) managing multiple sites in one license; and (4) risk-based reliance evaluation system. These four areas could be explored together with the feasible processes of how APEC economies could cooperate that would facilitate the defining or development of key performance indicators (KPIs) to measure the progress of convergence to assess whether "the regulatory requirements across economies become more similar or 'aligned' over time" (APEC RHSC Vision 2020). 


\section{Evolution of regulatory harmonization, convergence, and cooperation: The ICH experience}

From the early 1990s onwards, international regulatory harmonization for new drug development has been pursued under the efforts of $\mathrm{ICH}$. This has given rise to a standardized format, i.e. the common technical document (CTD), for companies to consolidate their scientific dataset for regulators' evaluation. Over the years, ICH members have also jointly developed some 70 guidelines to provide scientific principles and assessment details of the various sections listed in the CTD. Through the acceptance and full implementation of $\mathrm{ICH}$ guidelines by $\mathrm{ICH}$ members, regulatory requirements were harmonized among the ICH members. This great achievement has not only advanced access of new drugs to patients, but has also become the gold standard by which any regulatory authority can base its assessment framework for approving a new drug that is shown to be safe, efficacious and of good quality.

The ICH efforts have created a "common language" among international regulatory stakeholders and has promoted increasing cooperation among national regulatory authorities. Three levels of cooperation can generally be identified as shown in the shaded ellipses of Fig. 1 (Tominaga 2013):

Level 1: Information sharing/reliance.

Level 2: Working-sharing/mutual reliance.

Level 3: Mutual recognition.
Level 1 cooperation is feasible for regulators who adopt $\mathrm{ICH}$ requirements but are not yet full members of $\mathrm{ICH}$, and who can leverage on the assessments completed by stringent regulatory authorities (SRA) (WHO collaborative procedure 2017). The use of a SRA's assessment report enables a non- $\mathrm{ICH}$ regulator to expedite learning of $\mathrm{ICH}$ guidelines applied to evaluate scientific datasets and arriving at sound decisions while minimizing duplication efforts.

Level 2 cooperation takes place when the regulatory practice made on the data is also harmonized. For example, when confidence building is in place and the procedures for Good Manufacturing Practice (GMP) audit are harmonized, one authority can rely on the audit reports of another authority to base its decisions without duplicating the physical auditing process of the same manufacturing premises. This mutual reliance on work products of other authorities or work sharing among authorities is the next level of regulatory cooperation.

Level 3 and the highest level of regulatory cooperation is mutual recognition. However, the sharing of regulatory decisions among national regulatory authorities cannot be achieved easily due to factors such as differences in expertise and capacity, legal and risk frameworks, mutual understanding of systems and processes, and sovereignty issues. It is even more difficult when the process needs formalization and a legal arrangement such as a mutual recognition arrangement (MRA) must be concluded.

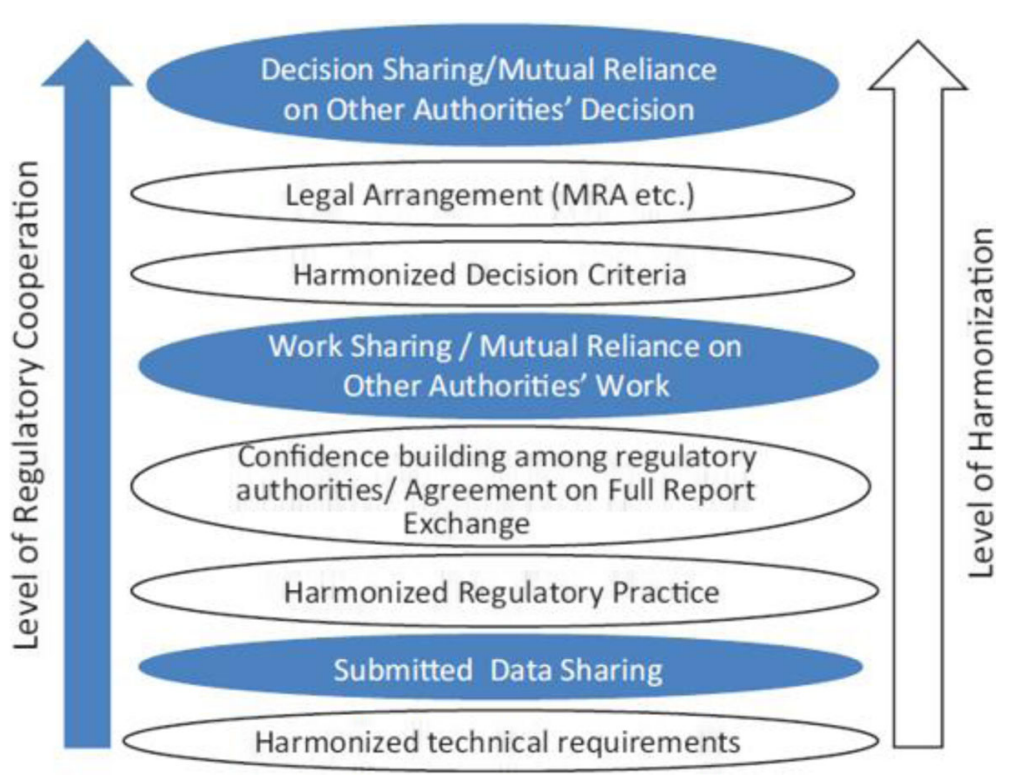

Fig. 1 (Tominaga 2013) Level of harmonization and regulatory cooperation. As the degree of regulatory cooperation advances from sharing of submitted data to work sharing/mutual reliance on other authorities' work, and to decision sharing/mutual reliance on other authorities' decision, the necessary level of harmonization also advances from that of technical requirements to regulatory practice and to decision criteria. Also necessary are other efforts such as confidence building and legal arrangements among the participating regulatory authorities 
The PIC/S established since 1995, is an extension to the Pharmaceutical Inspection Convention (PIC) of 1970. PIC/S is a non-binding co-operative agreement between regulatory authorities in the field of GMP of medicinal products for human or veterinary use. PIC/S aims at harmonizing the inspection procedures worldwide by developing common GMP standard and provide training opportunities to inspectors. It also aims to facilitate co-operation and networking between regulatory authorities (PIC/S Brochure 2016). The common standards and practices of $\mathrm{ICH}$ and $\mathrm{PIC} / \mathrm{S}$ requirements have facilitated the following examples of cooperation among $\mathrm{ICH}$ members:

(a) Information-sharing/reliance: Health Canada does not evaluate the drug master file of a generic drug if a Certificate of Suitability (CEP) issued by the European Directorate for the Quality of Medicines and Healthcare (EDQM) is already available (Government of Canada 2017);

(b) Work-sharing/mutual reliance: The U.S. Food and Drug Administration (USFDA) and European Medicines Agency (EMA) initiated their pilot joint assessment of Quality by Design dossier (EMA-FDA pilot program 2013);

(c) Mutual recognition: US FDA and EMA are currently developing a GMP MRA (EU-US mutual recognition of inspections 2017).

\section{Best practices and feasible processes for APEC economies' convergence}

In 2010, the APEC RHSC advocated for regulatory convergence in pharmaceuticals among its 21 economies so as to improve public health and economic development. The term "convergence" was clearly defined by the RHSC (APEC RHSC Vision 2020) with the understanding that:

- Regulatory convergence represents a voluntary process whereby the regulatory requirements across economies become more similar or "aligned" over time as a result of the gradual adoption of internationally recognized technical guidance documents, standards and scientific principles (harmonization) and common or similar practices and procedures.

- It does not represent the harmonization of laws and regulations, which is not necessary to allow for the alignment of technical requirements and for greater regulatory cooperation.

The RHSC initiative does not seek to develop new guidances; rather, it relies upon existing guidances already developed by international harmonization organizations, with $\mathrm{ICH}$ as the primary source. The efforts of RHSC focus on "convergence", i.e. on the process of aligning APEC economies' regulatory requirements based on best practices for optimal regulatory cooperation.

For the purposes of APEC RHSC activities and this Framework, the concept of "harmonization" represents the development and adoption of the same standards or requirements. Harmonization may also be applied to procedures and practices to see that these are the same across economies. The significance of the introduction of this concept is the emphasis that harmonization and convergence of drug regulations among multiple countries across the Asia-Pacific are ultimately for regulatory cooperation to expedite the access of patients to medicines and to benefit public health. In order for cooperation at the various levels (Fig. 1) to take place, regulatory processes and practices essential for such cooperation will first need to be put in place. However, while harmonization refers to processes that develop uniform standards across jurisdictions, regulatory convergence is a more pragmatic and feasible approach that better accommodates sovereignty and legal issues which make it difficult to achieve complete standardization across different economies.

This section aims to recommend four prioritized areas of appropriate regulatory practice in which convergence can be maximally achieved by 2020. Accordingly, KPIs can be developed in relation to these four areas to measure the progress of convergence to determine whether the regulatory requirements across economies become more aligned over time. The four areas are:

A. The appropriate use of the CPP;

B. The appropriate use of $\mathrm{PIC} / \mathrm{S}$ membership;

C. The appropriate management of multiple sites; and

D. The appropriate use of risk-based evaluation based on information

\section{(A) The appropriate use of the CPP}

The CPP was developed by the WHO as a tool to support product registration among national regulatory authorities, especially in developing countries (WHO Certification Scheme 2016). It serves as evidence that a product has been approved by the national regulatory authority that issued the CPP. The intent of the CPP is therefore to be used in lieu of full or partial review by the recipient country. As the original regulatory members of $\mathrm{ICH}$ conduct their reviews independently, they do not rely on the CPP approach for their decision-making. On the other hand, most of the APEC economies had adopted the use of CPP in their regulatory systems for product approval. Previously, Singapore (a member of both APEC and $\mathrm{ICH}$ ) used to request for 
CPPs but as its regulatory system matured, the requirement for the CPP has been removed from both its preand post-marketing control requirements.

With "convergence", the understanding is that "each economy may adopt each phase on its own timeframe" (APEC RHSC Vision 2020). In discussing a phased approach to achieve a maximum level of regulatory convergence by 2020, the 21 APEC economies may be broadly placed into 2 groups, namely those that depend on a CPP for regulatory decision making and those that do not.

Australia, Canada, Japan, Singapore and the United States of America (USA) are examples of non CPP-dependent APEC economies which do not require CPP for their product registrations. It is worth noting that these five examples are both regulatory members of $\mathrm{PIC} / \mathrm{S}$ and $\mathrm{ICH}$. Another observation is that various forms of cooperation already exist among these five APEC economies as that described in the preceding section. Such cooperation is facilitated by the common standards of $\mathrm{ICH}$ coupled with regulatory systems aligned with international practices implemented in their regulatory processes.

Among the APEC economies which are considered "CPP-dependent", China in recent years has undergone a series of regulatory reforms by converging its requirements towards international standards. One of the revisions China made in October 2017 is working towards the removal of CPP for Clinical Trial Application (CTA) and New Drug Application (NDA) applications for new chemical products and innovative biological products (CFDA Order No. 35 2017). It would therefore be useful for China to share with the other CPP-dependent APEC economies its rationale and approach for progressing from CPP-dependent to independent regulatory reviews based on ICH standards.

As the CPP is used to replace full or partial review of quality, safety and efficacy in line with WHO's advice, it is recommended that the need for CPP dependency amongst APEC economies that have already acquired PIC/S membership be reviewed, since these countries have already demonstrated capability to comply with international standards. It is therefore timely to review the relevance of CPP in both their pre- and post-marketing systems (WHO CPP Scheme 2015). However, for economies yet to achieve PIC/S membership, the CPP remains relevant in line with WHO advice.

\section{(B) The appropriate use of PIC/S membership}

The APEC economies that are PIC/S members could utilize the PIC/S network and common high standards to make significant positive moves towards greater convergence in GMP compliance assessment and conduct of inspections. It is recommended that these $\mathrm{PIC} / \mathrm{S}$ members streamline existing procedures so that:

(a) The current GMP related documentation required before inspections to support compliance assessment are minimized with country specific requirements such as unnecessary declaration forms, various reports and raw data, etc. (Nishimura 2017)

(b) Acceptance/reliance of PIC/S member inspection reports or GMP certification is in place so that inspection efforts are not unnecessarily duplicated (WHO Guidance on Good Practices 2017). Instead, on-site inspections should focus on manufacturing sites which are of concern, which have not historically been inspected by PIC/S inspectors, or have recently been noted to have GMP/quality concerns.

\section{(C) The appropriate management of multiple sites}

In October 2015, Chinese Taipei formally issued a new regulatory policy to allow a single product made in multiple sites to only have one license (Taiwan regulation 2015). Furthermore, when a new site is required to be added after its initial approval, a new license will not be warranted. Chinese Taipei changed its policy to align with international standards of regulating multi-sites in one license, and managing subsequent site additions as a post approval change, similar to what is practiced by ICH members.

The one-site-one-license process is commonly deployed among the APEC economies which are "CPP-dependent" (ASEAN Variation Guidelines 2013; Hong Kong Drug Office 2017), and this approach is quite the opposite of that practiced by ICH members. This results in highly duplicative and onerous procedures which are mainly administrative and have little (if no) positive impact on quality, safety and efficacy. The burden to apply and maintain multiple licenses for a single product significantly reduces product sourcing flexibility and affects continuity of supply, thus impacting patients in the case of product shortages. It is therefore strongly recommended that the APEC economies with the one-site-one-license requirement consider reviewing this system to allow for single licenses for multiple sites in order to converge regional regulatory practice in this particular area.

\section{(D) The appropriate use of risk-based evaluation based on information-sharing}

In today's regulatory landscape, public expectation is growing for maturing regulatory authorities to improve performance and facilitate timely access to safe, effective and quality products. This becomes more challenging as increasingly complex technologies and advanced 
therapies emerge. It is extremely important for regulatory authorities to prioritize optimal use of resources, while working towards attaining fully functional and effective regulatory systems according to international standards such as PIC/S. While working towards attaining PIC/S membership and to manage issues of scarce resources and expertise, APEC economies could consider adopting more risk-based approaches that leverage on evaluations conducted by stringent regulatory authorities, and focusing their regulatory resources on issues that are of locally critical significance. This will not only promote greater convergence in areas where appropriate scientific and technical assessments have already been completed by allied regulatory authorities but also help deploy expertise more efficiently (WHO Good Regulatory Practices 2016). An overview of Singapore's approach to risk-based evaluation that adopted this pragmatic approach to optimize limited resources effectively while maintaining robustness in its decisions constitutes a useful case study.

The Singapore Health Sciences Authority (HSA) attained PIC/S membership in 2000 (PIC/S members). This enabled HSA to be a member of a forum of established inspectors for networking and confidence building. For product evaluation, HSA leveraged on public assessment reports and stringent regulations of its five Reference Agencies (HSA Guidance (page 42 of 166) 2016) to approve products. Through the years of cooperation with these five agencies (involving memorandums of understanding, communication, training, information sharing, and staff attachments), HSA identified elements in the benefit-risk assessment that are more critical in the local context, and HSA was able to leverage the work of larger agencies while ensuring the benefit-risk assessments were applicable to the Singapore population (Patel et al. 2017). At the same time, the agency also implemented process reviews whereby its requirements become more aligned over time to established regulatory authorities as a result of the gradual adoption of internationally recognized guidance documents, scientific principles and best practices. It is of note that HSA eventually attained full membership of ICH in 2017 (HSA gains international acceptance 2017). This enabled Singapore to develop the necessary capabilities to perform full reviews for first-in-the-world products that had not been registered elsewhere (Wong \& Lim 2003). Capabilities to perform independent evaluation remain strategically critical and this ensures support for regional biomedical research and development growth population (Patel et al. 2017).

To ensure the effective use of resources, HSA also conducted a series of business process reviews (Haydock I 2010) to optimize productivity while ensuring robustness. This enabled HSA to rationalize what documents needed to be provided by the industry and which departments in the agency should evaluate the documents throughout the life cycle of the products. Pre- and post-marketing experts were organized into one common vigilance team to take charge of alert responses throughout the entire life-cycle of products, with activities including quality surveillance testing and pharmacovigilance monitoring. This streamlined the regulatory procedures to enable a seamless, integrated and science-based framework that is highly effective in risk mitigation, and also less bureaucratic in nature. To further increase efficiencies and avoid duplicative work, HSA introduced the "verification route" in 2004; this requires product approval by two or more reference agencies and HSA focuses on verifying the benefit-risk based on the assessment report of the selected reference agencies. This route has a short timeline of 60 working days (Patel et al. 2017).

On the basis of similarity in standard and procedures, a MRA on GMP conformity assessment between Australia and Singapore was signed on 26 February 2001. The agreement is of treaty status and includes the sector on Medicinal Product GMP Inspection. Under the MRA, the Therapeutic Goods Administration (TGA) of Australia accepts the conclusions of inspections of manufacturers carried out by GMP Auditors of HSA, Singapore and vice-versa. The GMP inspection report/ certificate is made available to the requesting party expeditiously, and the time taken should not exceed $30 \mathrm{cal}-$ endar days (HSA International Accreditation 2001). In 2011, two of Singapore's Reference Agencies, Health Canada and the TGA, invited Singapore to embark on a work sharing initiative for generics evaluation (International Consortium 2012). This highlights that Singapore is recognized as a credible partner in a four-member regulatory consortium that was established in 2007 (Australia-Canada-Singapore-Switzerland 2017).

This brief case study illustrates how the Singapore authority successfully achieved regulatory convergence by introducing sound risk-based approaches in its regulatory system through cooperation with selected international partners that it established Memorandums of Understanding (MOUs) and confidentiality agreements with. This provides important lessons on how relatively resource-strapped regulatory agencies can first leverage on stringent authorities' work results and later become aligned to the international standards and best practices that enable meaningful cooperation with stringent authorities to take place. The Singapore experience (Deloitte \& Touche LLP 2017; Lim 2016) can serve as a feasible model for other APEC economies as they develop strategies and procedures that leverage on assessments conducted by stringent regulatory authorities, in line with WHO's recommendation (WHO Good Regulatory Practices 2016; WHO collaborative procedure 2017). 


\section{Proposed KPIs to measure APEC regulatory convergence and cooperation}

Based on the discussion above, several KPIs linked to the four areas of appropriate regulatory practice could be used to help measure the progress of regulatory convergence on regulatory approval procedures for medical products across APEC economies by 2020 (Fig. 2).

The potential KPIs for the various economy groupings are as follows:

(a) APEC economies not yet a member of PIC/S:

(i) Number of economies with risk-based reliance evaluation system;

(ii) Number of economies attaining PIC/S status.

(b) APEC economies not yet a member of $\mathrm{ICH}$ :

(i) Number of economies removing CPP dependence and aligning with $\mathrm{ICH}$ practice;

(ii) Number of economies aligning GMP documentation and non-duplicative inspection to that of international practice;

(iii) Number of economies managing multi-sites in a single license. (c) APEC economies with membership of both PIC/S and $\mathrm{ICH}$ :

(i) Number of economies with work-sharing arrangements and mechanisms;

(ii) Number of economies with reliance/mutual recognition arrangements and mechanisms.

Regulatory convergence and cooperation should be promoted together. This is because for convergence to be meaningful, greater targeted cooperation amongst regulatory authorities should be realized in areas such as information sharing, relying on each other's decisions and work sharing to minimize duplication and enhance efficiency. Accordingly, the degree of success of regulatory convergence and cooperation will need to be measured by defining or developing a combination of KPIs that collectively measure convergence and cooperation.

\section{Capacity building}

To help promote convergence across the APEC region, capacity building is a clear need and enabler. Capacity building activities for regulatory authorities should be designed with clear awareness that one major purpose is for trainees to acquire skills and knowledge to enable

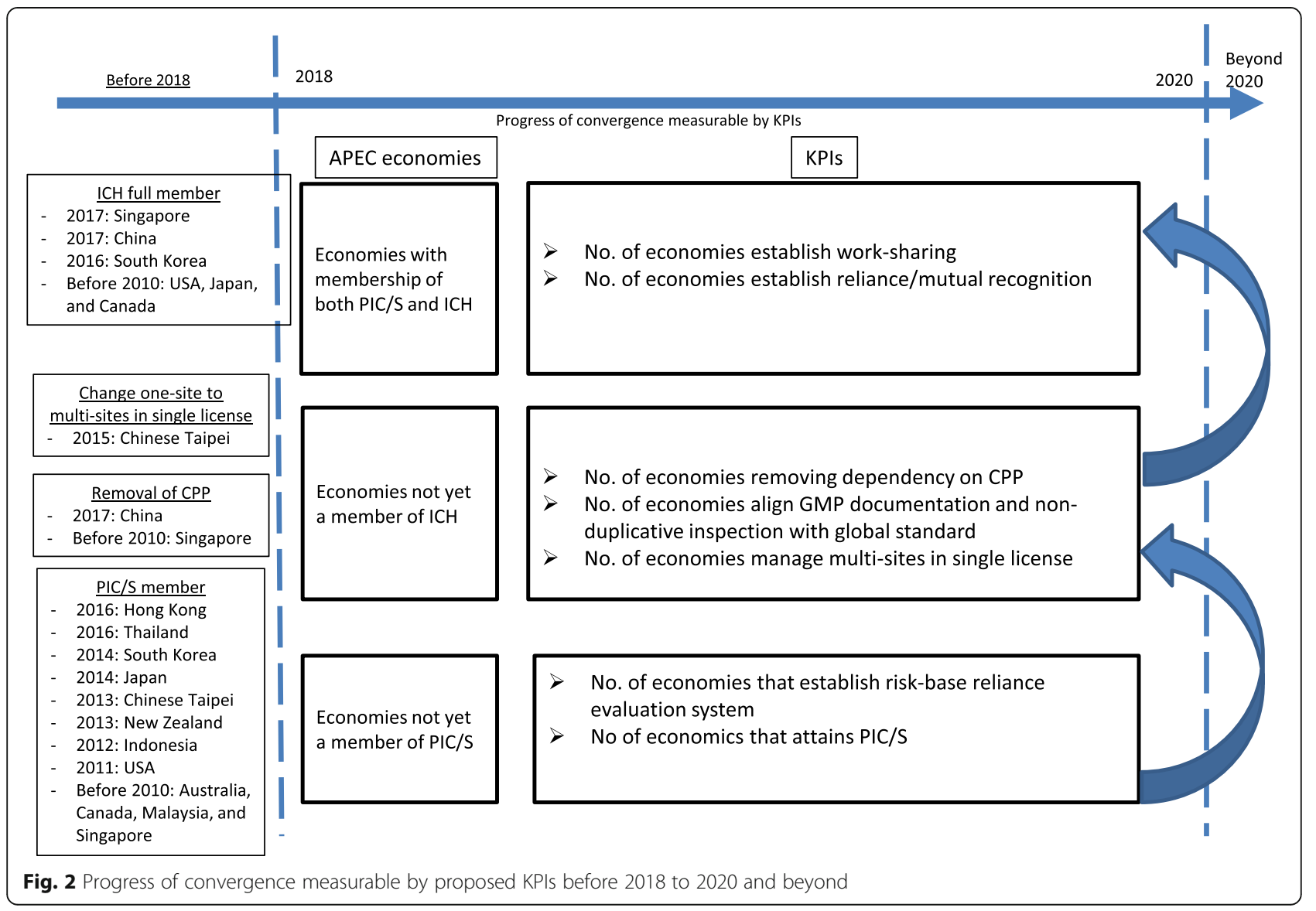


their organizations to start leveraging on stringent authorities' assessments and decisions in an appropriate manner, while developing their own regulatory capabilities to conduct independent, science-based reviews and other important local regulatory functions such as pharmacovigilance and supply chain integrity, in line with WHO's recommendation that regulatory authorities should focus "on what cannot be done by others while leveraging the work of other trusted NRAs and regulatory networks" (WHO Good Regulatory Practices 2016).

APEC has already introduced the frameworks for identifying and supporting Centres of Excellence (COEs) such as those in Duke-NUS Medical School in Singapore, Beijing University, Japan's Asia Training Center and Northwestern University in the USA (APEC Harmonization Center 2018). The courses of these COEs should aim to incorporate training on the use of public assessment reports or inspection reports issued by the stringent authorities to identify elements that matter in order to conduct appropriate benefit-risk assessments and reach sound regulatory decisions without compromising on scientific robustness. Trainees should be encouraged to develop risk-based reliance evaluation processes in their respective regulatory frameworks and become competent to eventually conduct independent evaluations based on international standards.

APEC economies can draw lessons from each other's experiences to expedite convergence through meaningful practical training that will bring about maximal outcomes. Based on the success stories of various APEC economies achieving convergence efforts cited in this paper, the following economies could potentially be invited to share experience in the context of existing or new CoEs:

(a) China - streamlining CPP dependency;

(b) Australia and Singapore - risk-based evaluation procedures based on information-sharing;

(c) Mexico - attaining PIC/S membership (Mexico to join PIC/S 2017);

(d) Canada, Australia and Singapore - minimizing country specific GMP documentation required as pre-inspection packages and non-duplicative inspection based on best practices (HSA Guidance Page 51-55, 2016);

(e) South Korea - becoming ICH member by the adoption of ICH Q1, Q7 and E6 guidelines (ICH Members \& Observers 2017);

(f) Chinese Taipei - managing multi-sites in one license.

\section{Conclusion}

National regulatory authorities are charged with the responsibility to ensure that the health products used by their populations are safe, efficacious and of good quality. It is also the duty of these authorities to expedite access of patients to the medicines and medical devices they need in a timely manner. With the ever increasing complexity of new biomedical and biotechnological products and development, coupled with the challenges arising from the global supply chain, the need for regulatory convergence and cooperation is essential rather than optional. In today's world, no single regulatory authority can claim that it is fully resourced to manage all issues relating to pre- and post-marketing of all the medicines and medical devices available in its country. The rational way forward is therefore to optimize the use of scarce resources in cooperation with regional and international regulatory counterparts, while administering a robust regulatory system that does not duplicate efforts to ensure timely access of health products to patients in a reliable and efficient manner.

APEC's continuing efforts to develop and promulgate good review practices are paving the way toward the sharing of review reports among participating authorities (Lin et al. 2015). Specifically, APEC regulators should embark on aligning best practices and processes of those prioritized areas as highlighted in this paper, as essential building blocks for ensuring the success of regulatory convergence.

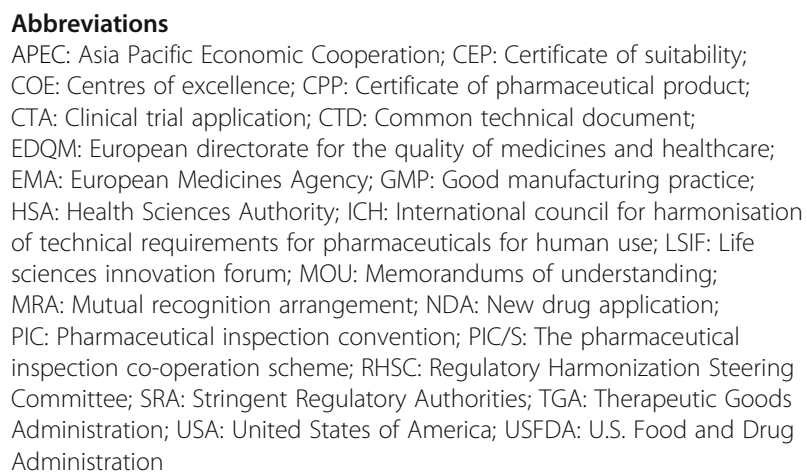

\section{Acknowledgements}

SSFC thanks Dr. Wassim Nashabeh from F. Hoffmann La-Roche Ltd. for his inspirational leadership in the advocacy of APEC regulatory convergence. The authors acknowledge Dr. Dorothy Toh from Singapore HSA for reviewing the case study of her organization, and Ms. Shamsuria Sumsoory for providing editorial support.

Funding

Processing fee of AAPS open was provided by SSFC's organization.

\section{Authors' contributions}

TT contributed to the writing on $\mathrm{ICH}$ and the creation of Fig. 1 with its level of convergence and cooperation. SSFC formulated the methodology with the four ideas of best practices identified, as well as the proposed KPIs to measure regulatory convergence. JCWL created the recommendation to incorporate sharing of the APEC economies' success into the existing CoEs as potential model for enhancing capacity building. Each author reviewed and approved the final version of the paper. 


\section{Competing interests}

The authors declares that they have no competing interests.

\section{Publisher's Note}

Springer Nature remains neutral with regard to jurisdictional claims in published maps and institutional affiliations.

\section{Author details}

1Asia Pacific Technical Regulatory Policy, Pharma Technical Regulatory Policy and International Operations, Roche Singapore Technical Operations, F. Hoffmann La-Roche Ltd, Singapore, Singapore. ${ }^{2}$ Centre of Regulatory Excellence, Duke-NUS Medical School, Singapore, Singapore.

${ }^{3}$ Pharmaceuticals and Medical Devices Agency (PMDA), Tokyo, Japan.

Received: 22 January 2018 Accepted: 23 April 2018

Published online: 31 May 2018

\section{References}

APEC Harmonization center (2018) Center of Excellence Overview http://www. nifds.go $. \mathrm{kr} / \mathrm{apec} /$ content/view.do? contentKey=288\&menuKey=318

APEC RHSC Vision 2020 (2010) A Strategic Framework: Regulatory Convergence for Medical Products by 2020. http://mddb.apec.org/documents/2011/MM/ AMM/11_amm_004att1.pdf

ASEAN Variation guidelines for pharmaceutical products (2013) http://www.hsa. gov.sg/content/dam/HSA/HPRG/Western_Medicine/Overview_Framework_ Policies/Guidelines_on_Drug_Registration/ASEAN\%20Variation\% 20Guideline\%20for\%20Pharmaceutical\%20Products\%207.2\%20clean\%20draft. pdf

Asia-Pacific Economic Cooperation (APEC), Member Economies (2017) https://www.apec.org/About-Us/About-APEC/Member-Economies

Australia-Canada-Singapore-Switzerland (ACSS) Consortium (2017) https://www. tga.gov.au/australia-canada-singapore-switzerland-acss-consortium

CFDA Order No. 35 (2017) "Decision on Adjusting the Management of Imported Drugs Registration" (October 2017). http://www.sfda.gov.cn/directory/web/ WS01/CL0053/178363.html

Deloitte \& Touche LLP (2017) Fostering Biomedical Innovation \& Excellence: Collaborating to enhance access to innovative medicines in Singapore

EMA-FDA pilot program for parallel assessment of Quality-by-Design applications: lessons learnt and Q\&A resulting from the first parallel assessment (2013) http://www.ema.europa.eu/docs/en_GB/document_library/Other/2013/08/ WC500148215.pdf

EU-US mutual recognition of inspections of medicines manufacturers enters operational phase (2017) http://www.ema.europa.eu/docs/en_GB/document_ library/Press_release/2017/10/WC500237909.pdf

Government of Canada (2017) Guidance Document: Use of Certificates of Suitability as supporting information in Drug Submissions https://www. canada.ca/en/health-canada/services/drugs-health-products/drug-products/ applications-submissions/quidance-documents/chemical-entity-productsquality/guidance-document-use-certificates-suitability-supportinginformation-drug-submissions.html

Haydock I (2010) Singapore looks beyond its shores. Regulatory Affairs J Pharma 21(4):229-230

Hong Kong Drug Office (2017) Guidelines on Application for Change of Registered Particulars of a Registered Pharmaceutical Product. http://www. drugoffice.gov.hk/eps/do/en/pharmaceutical_trade/guidelines_forms/ IcopGuide.html

HSA Therapeutic Products Guidance Page 42 of 166 (2016). http://www.hsa.gov sg/content/dam/HSA/HPRG/Western_Medicine/Overview_Framework_ Policies/Guidelines on Drug_Registration/DR Guide_2016/ DR\%20guide\%202016\%20final/Guidance\%20on\%20Therapeutic\%20Product\% 20Registration\%20in\%20Singapore\%202016.pdf

HSA Therapeutic Products Guidance Page 51-55 (2016). http://www.hsa.gov.sg/ content/dam/HSA/HPRG/Western_Medicine/Overview_Framework_Policies/ Guidelines_on_Drug_Registration/DR_Guide_2016/DR\%20guide\%202016\% 20final/Guidance\%20on\%20Therapeutic\%20Product\%20Registration\%20in\% 20Singapore\%202016.pdf

HSA gains international acceptance as a member of the ICH (2017). http://www. hsa.gov.sg/content/hsa/en/News_Events/Press_Releases/2017/hsa-gainsinternationalacceptanceasamemberoftheinternationalcoun.html

HSA International Accreditation, Liaisons and Agreements (2001) http://www.hsa. gov.sg/content/hsa/en/Health_Products_Regulation/Manufacturing_
Importation_Distribution/Overview/International_Accreditation_Liaisons_ and Agreements.html

ICH Members \& Observers (2017) http://www.ich.org/about/members-observers.html ICH Official website (2018) http://www.ich.org/home.html

International Consortium Aims to Facilitate Availability of Generic Drugs for Patients Through Focus on Generic Drug Review Collaboration (2012). http:// www.hsa.gov.sg/content/hsa/en/News_Events/HSA_Updates/2012/ international_consortium.html

Lim J-CW (co-author). Healthcare Regulation. In: Singapore's Health Care System - What 50 Years Have Achieved. World Scientific Publishing Co Pte Ltd; 2016. p 94-116. ISBN 9814696048

Lin H-Y, Chen S J-Y, Gau C-S, Liu L-L (2015) APEC Workshop Report of Good Review Practices on Medical Products, DIA Therapeutic Innovation \& Regulatory Science 49(4):483-492. DOl: https://doi.org/10.1177/ 2168479015572835. http://journals.sagepub.com/doi/pdf/10.1177/ 2168479015572835

Mexico to join PIC/S from January $1^{\text {st }} 2018$ (2017) https://www.picscheme.org/ en/news?itemid $=44$

Nishimura T (2017) Potential Areas for Improvement of GMP Compliance Assessment: Suggestion from Japan Pharmaceuticals Manufacturers Association (JPMA). Powerpoint presented at the $6^{\text {th }}$ Asia Partnership Conference of Pharmaceutical Associations (APAC). http://apac-asia.com/ achievements/6th_apac.html

Patel P, McAuslane N, Liberti L, Connelly P (2017) Facilitating the review of new medicines through risk-based evaluations: How can a stratification process be utilised to achieve an effective use of resources, Workshop Report, Centre for Innovation in Regulatory Science (CIRS)

PIC/S Brochure 2016, https://www.picscheme.org/layout/document.php?id=692. $\mathrm{PIC/S}$ Members Official website. https://www.picscheme.org/en/members

Taiwan regulation. http://regulation.cde.org.tw/. Notice No. 1041409729 (2015). http://regulation.cde.org.tw/doc_data_display?sid=2398\&doctype2=

Tominaga T (2013) The ICH, the GHTF, and the future of harmonization initiatives. Therapeutics Innov Regul Sci 47:572

WHO CPP Scheme in today's regulatory environment - is it time for change (2015). WHO drug information Vol. 29, No 4

WHO Certification Scheme on the quality of pharmaceutical products moving in international commerce: Questions and Answers (2016). WHO Drug Information Vol. 30, No. 3

WHO collaborative procedure in the assessment and accelerated national registration of pharmaceutical products approved by stringent regulatory authorities (2017)

WHO Guidance on Good Practices for desk assessment for compliance with Good Manufacturing Practices, Good Laboratory Practices and Good Clinical Practices for Marketing Authorization of Medical Products (2017)

WHO Good Regulatory Practices (2016) Guidelines for national regulatory authorities for medical products

Wong G- BN, Lim J-CW (2003) Drug regulation by Singapore's health sciences authority: the role of the Centre for Drug Evaluation. Drug Info J 37:15(S)25(S) 2003 0092-8615/2003

\section{Submit your manuscript to a SpringerOpen ${ }^{\mathcal{O}}$ journal and benefit from:}

- Convenient online submission

- Rigorous peer review

- Open access: articles freely available online

- High visibility within the field

- Retaining the copyright to your article

Submit your next manuscript at springeropen.com 